\title{
Symmetry Induced Half-Metallic Alkaline Earth Ferromagnets
}

\author{
L. AdAmowicz And M. WierzBicki \\ Faculty of Physics, Warsaw University of Technology \\ Koszykowa 75, 00-662 Warszawa, Poland
}

\begin{abstract}
Search for new half-metallic ferromagnetic binary compounds composed of alkaline earth and III (boron) or IV (carbon) elements is reported. Ab initio all-electron density functional theory calculations in the generalised gradient approximation indicate possibility of half-metallic ferromagnetism. This is a new class of theoretically predicted hypothetical materials, without transition metal elements, not as yet discovered experimentally. Ferromagnetism is expected to be induced by suitable crystalline structure with lattice constant above some critical value. The predominant $p$ electrons mechanism seems to be responsible for the formation and interactions of localised magnetic moments on boron or carbon atoms.
\end{abstract}

PACS numbers: 71.15.Mb, 71.20.Dg, 72.25.Ba, 75.10.Lp

\section{Introduction}

Recently, an unusual class of ferromagnetic materials [1-3] which do not contain transition-metal or rare-earth atoms was proposed and analysed theoretically by $a b$ initio calculations. The predictive power of these calculations has been successfully used to investigate the properties of binary compounds like $\mathrm{MgC}, \mathrm{CaC}, \mathrm{SrC}$ and $\mathrm{BaC}$ [4]. Using the first-principles full-potential linearised augmented plane-wave method based on the density functional theory, the electronic structure and magnetism of these compounds in the zinc-blende (ZB) structure have been studied. Surprisingly enough, for $\mathrm{CaC}$, $\mathrm{SrC}$ and $\mathrm{BaC}$ the minority-spin electrons are metallic, whereas the majority-spin electrons are described by the energy band lying well below Fermi level and separated from it by an energy gap of few electronvolts. Therefore these three compounds in zinc-blende crystalline structure are half-metallic (HM) ferromagnets of similar character. In particular, their calculated total magnetic moments per formula unit are identical and equal to $2.00 \mu_{\mathrm{B}}$. The main contribution to the magnetic moment comes from electrons on the $\mathrm{C}$ anion. The total magnetic moment remains integer until the lattice constant is compressed to the critical value [4]. The estimated contraction in the range from $9 \%(\mathrm{BaC})$ to $14 \%$ (for $\mathrm{CaC}$ ) for $\mathrm{ZB}$ structure makes these HM materials promising for epitaxial growth and subsequent spintronic and other magnetoelectronic applications [5].

Recently, Chang-wen Zhang et al. [6] have investigated HM ferromagnetism in wurtzite (WZ) SrC. They calculated total and partial density of states (DOS) with optimised lattice constant in the generalised gradient approximation (GGA) for the exchange-correlation po- tential within the framework of density functional theory (DFT). The minority-spin electrons are metallic. The DOS around the Fermi level mainly originates from Sr $d$ and $\mathrm{C} p$ electron states. The valence band is almost composed of $\mathrm{C} p$ electrons, but the dispersion of the top band is determined by $p-d$ hybridisation. The top of the majority-spin valence band is at $0.72 \mathrm{eV}$ below the Fermi level. This energy of $0.72 \mathrm{eV}$ needed to activate majority-spin channel shows that $\mathrm{SrC}$ in $\mathrm{WZ}$ structure is also HM ferromagnet. Results [6] of the calculations clearly demonstrate that $\mathrm{WZ} \mathrm{SrC}$ is a robust HM ferromagnet with magnetic moment of $2 \mu_{\mathrm{B}}$ per formula unit. The half-metallicity is maintained up to the lattice compression of $21 \%$. Therefore, one may expect to grow $\mathrm{SrC}$ on an appropriate substrate in the form of a film or even a bulk sample.

In this paper we present $a b$ initio calculations, motivated by the described above findings $[4,6]$. We concentrate on $\mathrm{BaC}$ and $\mathrm{BaB}$ in $\mathrm{WZ}$ structure. The aim of this study is to verify the general features and mechanisms [2] leading to HM ferromagnetism without presence of transition-metal elements. Results of the calculations, presented in the next section, show that $\mathrm{WZ} \mathrm{BaC}$ is $\mathrm{HM}$ ferromagnet with magnetic moment of $2 \mu_{\mathrm{B}}$ per formula unit. Similar result was obtained in the case of $\mathrm{WZ} \mathrm{BaB}$, but with the magnetic moment of $3 \mu_{\mathrm{B}}$ per formula unit. Details of the method are given in the next section. Discussion of the results and concluding remarks are given in the last section of the paper.

\section{Method}

The $a b$ initio all electron DFT calculations have been performed for $\mathrm{BaC}$ and $\mathrm{BaB}$ in $\mathrm{WZ}$ structure in the lo- 


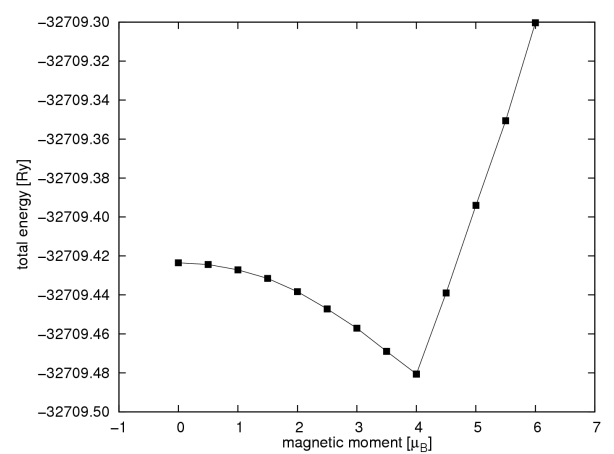

Fig. 1. The total energy as a function of magnetic moment per unit cell for $\mathrm{BaC}$ in wurtzite structure with $a=4.41 \AA$ and $c=7.92 \AA$. The fixed-spin-moment option of the WIEN2k package to constrain the total magnetic moment per unit cell to a fixed value was used. Minimum energy magnetic moment of $4 \mu_{\mathrm{B}}$ corresponds to $2 \mu_{\mathrm{B}}$ per formula unit.

cal spin density approximation (LSDA) by adding gradient terms of the electron density to the exchange-correlation potential. This GGA approximation is used in the Perdew-Burke-Ernzerhof [7] parameterisation. It should be mentioned that LSDA gives the magnetic moments in very good agreement with experiment but underestimates the lattice constants in the case of transition metals. On the other hand, gradient corrections significantly reduce this error and give the correct phase stability but tend to overestimate the magnetic moment. We did not check this deficiency of GGA in our calculations and assumed that it has a small effect on the considered systems. The full potential linearised augmented plane wave method with the scalar relativistic treatment of the core electrons, as implemented in the WIEN2k package [8], was used. The convergence of the basis set was controlled by the cut-off parameter $\mathrm{RK}_{\max }=8$ together with $2000 k$-point mesh for the integration over the Brillouin zone. The angular momentum expansion up to $l=10$ for the potential and charge density was employed in the calculations. Self-consistency was considered to be achieved when the total energy difference between succeeding iterations is smaller than $10^{-5} \mathrm{Ry}$ per formula unit. Geometry optimisation was performed allowing all atoms in the unit cell to relax, constrained to the initially assumed crystal symmetry.

\section{Results and discussion}

In order to investigate the properties of $\mathrm{BaC}$ and $\mathrm{BaB}$ in WZ structure we first optimised lattice constants from the minimum of the total energy. For $\mathrm{BaC}$ we find $a=4.41 \AA$ and $c=7.92 \AA$. The obtained global magnetic moment of $4 \mu_{\mathrm{B}}$ per unit cell $\left(2 \mu_{\mathrm{B}}\right.$ per formula unit) demonstrates ferromagnetic ordering of the sample (Fig. 1).

The calculated spin-dependent band structure is illustrated in Fig. 2 along the selected high-symmetry lines in

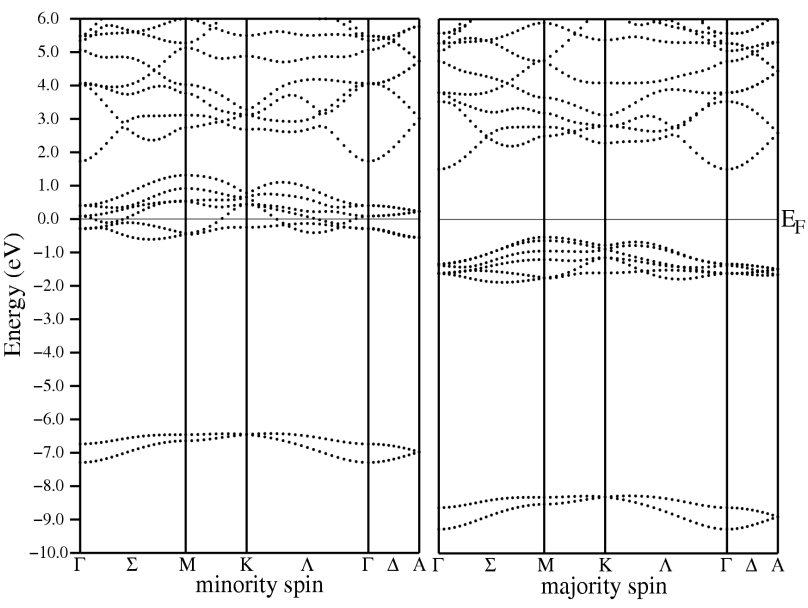

Fig. 2. Band structure diagram of wurtzite $\mathrm{BaC}$ with $a=4.41 \AA$ and $c=7.92 \AA$. The path in the Brillouin zone is as for a hcp structure. All energies are relative to the Fermi level. Half-metallic energy gap of $0.53 \mathrm{eV}$ for majority spin states can be seen in the right part.

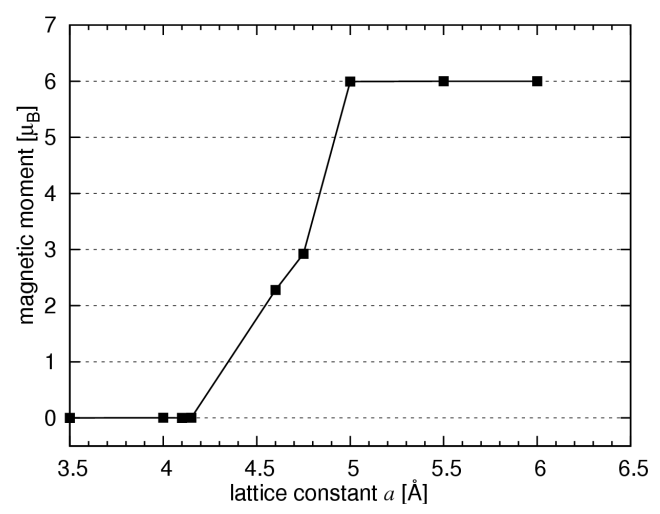

Fig. 3. Total magnetic moment as a function of lattice constant $a$ for $\mathrm{BaB}$ in wurtzite structure with ideal ratio $c / a=\sqrt{8 / 3}$. Half-metallicity is maintained up to the value $a=5 \AA$ in lattice compression.

the Brillouin zone of $\mathrm{WZ} \mathrm{BaC}$. The $s$ states of $\mathrm{C}$ lie very low in energies near $-9 \mathrm{eV}$ and are well separated from other bands. The other six bands in the vicinity of the Fermi level mainly originate from carbon $p$ and barium $d$ states, which can be easily deduced from the partial DOS. As in the case of $\mathrm{SrC}[6]$ the $p$ states of carbon are strongly localised on $\mathrm{C}$ atom and hybridisation with the $d$ states of $\mathrm{Ba}$ is rather weak, leading to flat dispersionless bands near the Fermi level. For majority spin states these bands are completely filled, while for the minority spin states they are cut by the Fermi level and thus partially occupied. As a consequence, resulting magnetic moment of $2 \mu_{\mathrm{B}}$ per formula unit is created with only one (minority) active spin channel. The HM energy gap of $0.53 \mathrm{eV}$ prevents from activating majority spin channel. As in the case of other HM ferromagnets, like $\mathrm{CaC}$ and $\mathrm{SrC}[4,6]$, the carbon anion is the main contributor to 


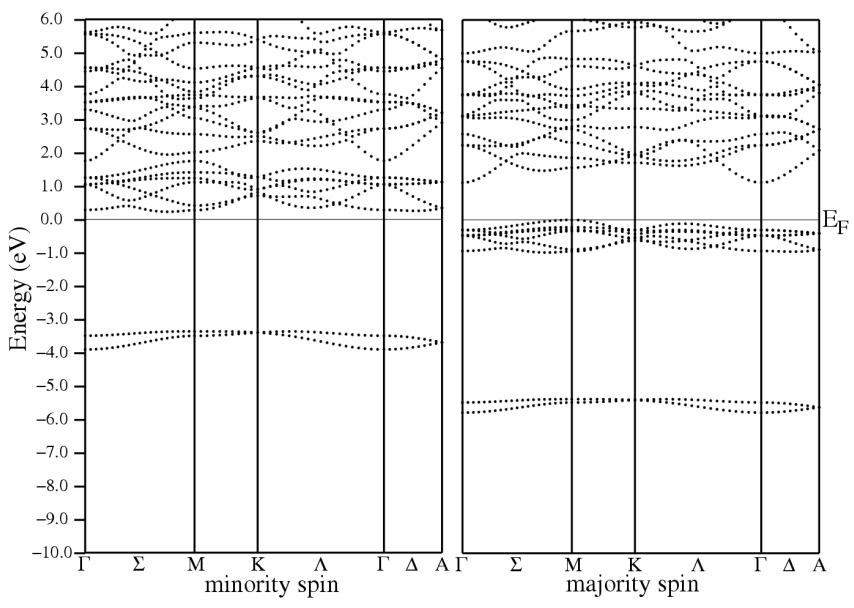

Fig. 4. Band structure diagram of $\mathrm{BaB}$ in ideal wurtzite structure with $a=5.5 \AA$. The path in the Brillouin zone is as for a hcp structure. All energies are relative to the Fermi level. Half-metallic energy gap of $0.24 \mathrm{eV}$, visible in the left part, separates majority and minority spin states, as can be seen by comparing both parts.

the magnetic moment. The half-metallicity is attributed to the $p-d$ hybridisation ruled by the WZ symmetry and leading to the bonding-antibonding splitting. This contrasts with the ZB transition-metal pnictides and chalcogenides, where the anion $p$ states are completely filled and the magnetic moment is mainly formed by the $3 d$ electron states. Since $\mathrm{BaC}$ does not appear in nature one may think of $\mathrm{WZ}$ phase of $\mathrm{BaC}$ as a useful material for epitaxial growth of films on a proper substrate of WZ semiconductor.

Replacement of $\mathrm{C}$ by $\mathrm{B}$ in $\mathrm{BaC}$ leads to $\mathrm{WZ} \mathrm{BaB}$. Results of calculations are shown in Figs. 3 and 4 . Since the lattice parameter of an epitaxially grown film de- pends on the lattice constant of the substrate it is important to know the robustness of the half-metallicity with respect to the variation of the interatomic distance. The total magnetic moment remains integer until the lattice constant is compressed to the critical value of $5 \AA$ (Fig. 3). The estimated contraction in the range of $17 \%$ makes WZ BaB a promising material for device applications. Band structure diagram of $\mathrm{BaB}$ (Fig. 4) in ideal wurtzite structure with $a=5.5 \AA$ indicates three substantial changes as compared to BaC. First, the global magnetic moment has increased to $6 \mu_{\mathrm{B}}$ per unit cell (Fig. 3) or $3 \mu_{\mathrm{B}}$ per formula unit. Second, the half-metallic gap has decreased to the value of $0.24 \mathrm{eV}$. Third, as is seen from Fig. 4, the half-metallicity is maintained by the majority spin channel accompanied by the empty minority spin bands. In conclusion, replacement of carbon by boron produced the decrease in the number of valence electrons (per formula unit) by one and subsequent creation of local magnetic moment of $3 \mu_{\mathrm{B}}$ on $\mathrm{B}$, conserving the general mechanism of half-metallicity described in earlier publications $[1-4,6]$.

\section{References}

[1] K. Kusakabe, M. Geshi, H. Tsukamoto, N. Suzuki, J. Phys., Condens. Matter 16, S5639 (2004).

[2] M. Sieberer, J. Redinger, S. Khmelevskyi, P. Mohn, Phys. Rev. B 73, 024404 (2006).

[3] O. Volnianska, P. Bogusławski, Phys. Rev. B 75, 224418 (2007).

[4] G.Y. Gao, K.L. Yao, E. Şaşığlu, L.M. Sandratskii, Z.L. Liu, J.L. Jiang, Phys. Rev. B 75, 174442 (2007).

[5] I. Žutić, J. Fabian, S. Das Sarma, Rev. Mod. Phys. 76, 323 (2004).

[6] Chang-wen Zhang, Shi-shen Yan, Hua Li, Phys. Status Solidi B 245, 201 (2008).

[7] J.P. Perdew, K. Burke, M. Ernzerhof, Phys. Rev. Lett. 77, 3865 (1996).

[8] P. Blaha, K. Schwarz, G.K.H. Madsen, D. Kvasnicka, J. Luitz, in: WIEN2k, An Augmented Plane Wave Plus Local Orbitals Program for Calculating Crystal Properties, Ed. K. Schwarz, Techn. Universität Wien, Austria 2001 